\title{
Risk factors for synchronous or metachronous tumor development after endoscopic resection of gastric neoplasms
}

\author{
Joo Hyun Lim $\cdot$ Sang Gyun Kim $\cdot$ Jeongmin Choi $\cdot$ \\ Jong Pil Im • Joo Sung Kim $\cdot$ Hyun Chae Jung
}

Received: 31 July 2014/ Accepted: 5 October 2014/Published online: 18 October 2014

(c) The International Gastric Cancer Association and The Japanese Gastric Cancer Association 2014

\begin{abstract}
Background Despite many advantages, the development of synchronous or metachronous neoplasm is one of the main concerns with endoscopic resection. We aimed to clarify the independent risk factors for synchronous or metachronous gastric neoplasm.

Methods We retrospectively reviewed the medical records of all patients who had undergone endoscopic resection for gastric high-grade dysplasia or early gastric cancer between April 2001 and February 2011.

Results Among 971 subjects, 56 synchronous neoplasms and 42 metachronous neoplasms developed during 12-131 months of follow-up. In univariate analysis, age over 65 years, male gender, absence of Helicobacter pylori infection, lower third location, mucosal atrophy, and intestinal metaplasia were related to multiple gastric neoplasms. In multivariate analysis, absence of $H$. pylori infection [odds ratio (OR) 1.610, $95 \%$ confidence interval (CI) 1.038-2.497)], lower third location (OR 1.704, $95 \%$ CI 1.070-2.713), and intestinal metaplasia (OR 4.461, $95 \%$ CI 1.382-14.401) were independent risk factors for multiple gastric neoplasms. For synchronous neoplasm, primary tumor size less than $1 \mathrm{~cm}$ was the only
\end{abstract}

The abstract of this study was presented at United European Gastroenterology Week 2012 in the "Top Late Breaking Abstracts in GI/Liver Cancer" session as an oral presentation and at Digestive Disease Week 2013 as a poster.

J. H. Lim · S. G. Kim (凹) · J. Choi · J. P. Im •

J. S. Kim · H. C. Jung

Division of Gastroenterology, Department of Internal Medicine and Liver Research Institute, Seoul National University College of Medicine, Yongun-dong 28, Chongno-gu, Seoul 110-744, South Korea

e-mail: harley1333@hanmail.net independent risk factor. For metachronous neoplasm, absence of $H$. pylori infection (OR 2.416, $95 \%$ CI 1.214-4.810) was found to be the only independent risk factor. H. pylori eradication was found to be unrelated to the development of metachronous gastric neoplasms.

Conclusions For tumors located in the antrum and accompanied by intestinal metaplasia, meticulous endoscopic evaluation with close follow-up after endoscopic resection is recommended.

Keywords Endoscopic resection - Precancerous conditions and cancerous lesions - Gastrointestinal disease

\section{Introduction}

Endoscopic resection has been widely used as curative treatment of gastric tumors, which preserves the stomach and allows radical resection. Especially, endoscopic submucosal dissection (ESD) allows en bloc resection with tumor-free margins, even in cases with large and ulcerative lesions [1, 2]. According to previous reports, the complete en bloc resection rate of ESD was over $80 \%$, which was significantly higher than that of conventional endoscopic mucosal resection [3]. Despite its convenience, there is a major concern about the high rate of newly developed neoplasms after endoscopic resection in the remaining gastric mucosa. In previous studies, the incidence of synchronous or metachronous gastric cancer after surgical resection was reported to range from as high as 4.8-20.9\% [4-9] to as low as $1.8-2.4 \%[10,11]$. In another study, the incidences of synchronous and metachronous gastric cancer after endoscopic resection were 11 and $14 \%$, respectively [12].

There have been several studies suggesting possible predictive factors for metachronous gastric cancer. In one 
study, metachronous gastric cancer tended to develop in the same third of the stomach as the primary lesion with similar macroscopic and differentiated type [12]. Another two studies showed that atrophy in the background gastric mucosa was associated with the development of metachronous gastric cancer $[13,14]$. In another study, undifferentiated histologic appearance of the primary lesion was related to the occurrence of synchronous or metachronous gastric cancer [15]. Helicobacter pylori infection is also considered to be a major risk factor for gastric cancer; however, there are still many debates about whether this is an independent risk factor for the development of metachronous gastric cancer [16, 17]. However, all these studies enrolled relatively small numbers of subjects and had short follow-up periods. Also, as high-grade dysplasia is considered as a premalignant lesion, it seems reasonable to include these premalignant lesions to evaluate the risk of synchronous or metachronous gastric neoplasms.

Therefore, this study was designed to clarify the independent risk factors for synchronous or metachronous gastric neoplasm development after endoscopic resection of gastric high-grade dysplasia and early gastric cancer (EGC) with a large number of subjects enrolled.

\section{Materials and methods}

\section{Patients}

A total of 1,889 patients had undergone ESD or endoscopic mucosal resection for gastric neoplasms at Seoul National University Hospital between April 2001 and February 2011. Endoscopic resection was performed for lesions with the following criteria: any lesions of low-to-high grade dysplasia regardless of size, or well-to-moderately differentiated adenocarcinoma confined to mucosa less than $2 \mathrm{~cm}$ in diameter by endoscopic measurements without evidence of lymph nodal/distant metastases on abdominal computerized tomography/endoscopic ultrasonography. Pre-ESD size measurement was performed macroscopically in comparison with fully open forceps. For the initial diagnosis, two pieces of mucosa were biopsied. After complete endoscopic resection for gastric neoplasms, follow-up endoscopy was scheduled at the third, sixth, and twelfth months and thereafter every year.

High-grade dysplasia was defined as an intraepithelial neoplasia of category 4 in the revised Vienna classification including epithelial dysplasia regardless of mucosal elevation, and EGC was defined as an invasive carcinoma of category 5 [18]. Undifferentiated histologic appearance was defined as a tumor in which more than $50 \%$ of the tumor area contains poorly differentiated, signet ring cell, poorly cohesive, or mucinous carcinoma.
The status of $H$. pylori infection was evaluated by using the rapid urease test at the antrum and histologic examination at the antrum and body. Infection was considered to be present if either of the tests gave a positive result. Intestinal metaplasia or mucosal atrophy was considered to be present if the histologic examination of the biopsy sample taken from the antrum and body revealed mild or higher-grade intestinal metaplasia or mucosal atrophy in the Sydney classification.

This study was approved by the Institutional Review Board of Seoul National University Hospital. Patient consent was waived, given the retrospective nature of this study.

Definition of synchronous and metachronous gastric neoplasm

In this study, synchronous neoplasm was defined as a newly developed high-grade dysplasia or adenocarcinoma at another site in the stomach within 1 year after endoscopic resection for the primary lesion or a simultaneously existing lesion. Metachronous neoplasm was defined as a tumor which had developed more than 1 year after the primary procedure.

\section{Data analysis}

All subjects were grouped into one of the following groups: single neoplasm group, synchronous neoplasm group, and metachronous neoplasm group. Patients with multiple synchronous and/or metachronous tumors detected during the follow-up periods were assigned as to their first new lesions, and for these patients only the first new lesions were investigated. For patients with multiple lesions detected simultaneously, the most advanced lesion was counted as the primary one.

To search for potential risk factors that influence the development of multiple neoplastic lesions, the following variables were analyzed; age (younger than 65 years, or 65 years or older), gender, H. pylori status, pathologic diagnosis (high-grade dysplasia or EGC), tumor differentiation (well-to-moderately differentiated or undifferentiated), depth of tumor invasion (mucosa or submucosa), lymphovascular invasion, tumor size (less than $1 \mathrm{~cm}$, or $1 \mathrm{~cm}$ or larger), location (upper, middle, or lower third) of the primary lesion, atrophy, and intestinal metaplasia in the background gastric mucosa.

Statistical analysis

Categorical variables were analyzed with the chi square test and Fisher's exact test for univariate analysis. Those variables with $p<0.200$ in the univariate analyses were 
examined in multivariate binary logistic regression models. We considered $p<0.050$ to be significant. All $p$ values are presented uncorrected for multiple testing. All of the analyses were performed with use of Statistical Package for the Social Sciences, version 20.0 for Windows (SPSS, Chicago, IL, USA).

\section{Results}

Among the 1,889 cases of endoscopic resections, 713 patients with low-grade dysplasia were excluded because of its low malignant potential, and 135 patients were excluded because of less than 1 year of follow-up. Seventy patients were excluded because of additional gastrectomy for noncurative ESD. Therefore, a total of 971 subjects, including 184 patients with high-grade dysplasia and 787 patients with EGC, were reviewed retrospectively (Fig. 1). Among a total of 971 subjects, 873 had single neoplasms and 98 had multiple neoplasms (56 synchronous lesions and 42 metachronous lesions). Among the 56 synchronous neoplasms, 33 were detected simultaneously with the primary lesions. The cumulative incidence of metachonous neoplasm was 1.4 per 100 person-years.

\section{Baseline characteristics}

Overall, the mean age was 63 years and the proportion of males was $74 \%$ (Table 1). The rate of $H$. pylori infection was $55 \%$. More than $80 \%$ of the primary lesions were adenocarcinomas. The mean diameter of the primary lesions was $1.9 \mathrm{~cm}$. Most of the lesions were located in the lower third of the stomach, with a rate of $61 \%$, whereas in the middle third and upper third the rates were 30 and $9 \%$, respectively. Atrophic gastritis was found in $70 \%$ of patients and intestinal metaplasia was found in $88 \%$ of patients. Undifferentiated histologic appearance, submucosal invasion, and lymphovascular invasion were detected in 9.6, 8.8 and $1.8 \%$ of patients, respectively. The mean follow-up duration was $37.1 \pm 19.3$ months (range 12-131 months).

Among the three groups, the mean age was significantly higher in the synchronous and metachronous neoplasm groups $(p=0.019)$. However, H. pylori infection was less common in the synchronous and metachronous neoplasm groups than in the single neoplasm group $(p=0.011)$. Intestinal metaplasia was more prevalent among the multiple neoplasm groups $(p=0.018)$.

Risk factors for multiple gastric neoplasms

Univariate analysis for multiple neoplasms showed that age over 65 years, male gender, $H$. pylori infection, lower third location, gastric atrophy, and intestinal metaplasia were related to multiple gastric neoplasms (Table 2). Among these factors, H. pylori infection exhibited an inverse relationship with multiple neoplasms. Multivariate analysis with these variables revealed that the absence of $H$. pylori infection [odds ratio (OR) 1.610; $p=0.033$ ], lower third location (OR 1.610; $p=0.046)$, and intestinal metaplasia (OR 4.321; $p=0.014$ ) were the independent risk factors for multiple neoplasms (Table 3).

The H. pylori prevalence was $61.1 \%(312 / 511)$ among those younger than 65 years, and $47.4 \%$ (200/422) among those aged 65 years or older. Most of the $H$. pylori-negative patients did not recall whether they had had previous eradication therapy or not. Among the patients with H. pylori infection, 236 received eradication therapy and 276 did not. Among those with eradication therapy, metachronous gastric
Fig. 1 A total of 971 subjects, including 184 high-grade dysplasia patients and 787 early gastric cancer patients. EMR endoscopic mucosal resection, ESD endoscopic submucosal dissection

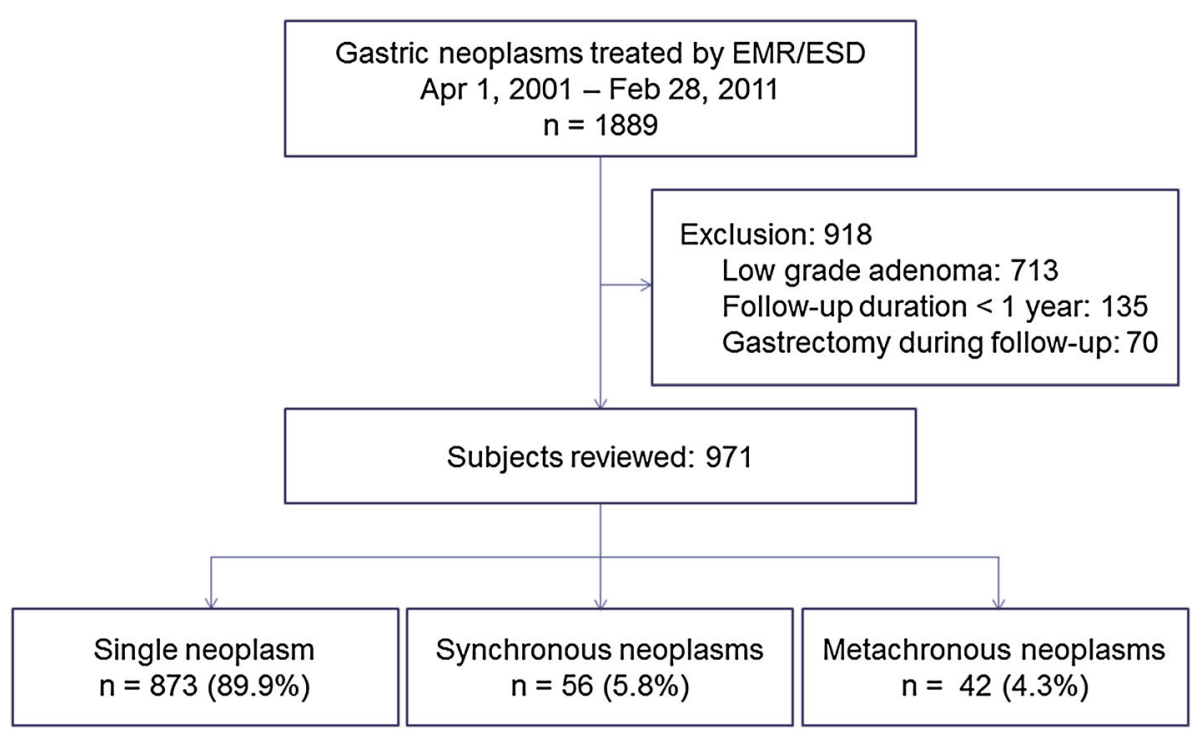


Table 1 Baseline characteristics

\begin{tabular}{|c|c|c|c|c|c|}
\hline & $\begin{array}{l}\text { Overall } \\
(n=971)^{\mathrm{b}}\end{array}$ & $\begin{array}{l}\text { Single neoplasm } \\
(n=873)^{\mathrm{b}}\end{array}$ & $\begin{array}{l}\text { Synchronous } \\
\text { neoplasm }(n=56)^{\mathrm{b}}\end{array}$ & $\begin{array}{l}\text { Metachronous } \\
\text { neoplasm }(n=42)^{\mathrm{b}}\end{array}$ & $p^{\mathrm{c}}$ \\
\hline Mean age (years) ${ }^{\mathrm{a}}$ & $63.05(9.508)$ & $62.79(9.631)$ & $64.57(7.813)$ & $66.60(8.166)$ & 0.019 \\
\hline Male gender $(\%)$ & $721 / 971(74.3)$ & $640 / 873(73.3)$ & $44 / 56(78.6)$ & $37 / 42(88.1)$ & 0.076 \\
\hline Helicobacter pylori positive $(\%)$ & $512 / 933(54.9)$ & $471 / 838(56.2)$ & $28 / 55(50.9)$ & $13 / 40(32.5)$ & 0.011 \\
\hline Carcinoma $(\%)$ & $787 / 971(81.1)$ & $704 / 873(80.6)$ & $50 / 56(89.3)$ & $33 / 42(78.6)$ & 0.255 \\
\hline Mean size $(\mathrm{cm})^{\mathrm{a}}$ & $1.862(1.208)$ & $1.863(1.216)$ & $1.720(1.169)$ & $2.027(1.079)$ & 0.463 \\
\hline Location & & & & & 0.299 \\
\hline Upper third (\%) & $87 / 971(9.0)$ & $83 / 873(9.5)$ & $2 / 56(3.6)$ & $2 / 42(4.8)$ & \\
\hline Middle third (\%) & 292/971 (30.1) & $262 / 873(30.5)$ & $16 / 56(28.6)$ & $10 / 42(23.8)$ & \\
\hline Lower third $(\%)$ & $592 / 971(61.7)$ & $524 / 873(60.0)$ & $38 / 56(67.9)$ & $30 / 42(71.4)$ & \\
\hline Atrophy (\%) & $524 / 753(69.6)$ & $470 / 683(68.8)$ & $33 / 43(76.7)$ & $21 / 27(77.8)$ & 0.352 \\
\hline $\mathrm{IM}(\%)$ & $819 / 932(87.9)$ & $727 / 837(86.9)$ & $53 / 55(96.4)$ & $39 / 40(97.5)$ & 0.018 \\
\hline Undifferentiated $(\%)$ & 93/970 (9.6) & $87 / 872(10.0)$ & $4 / 56(7.1)$ & $2 / 42(4.8)$ & 0.435 \\
\hline SM invasion (\%) & $85 / 970(8.8)$ & $75 / 872(8.6)$ & $7 / 56(12.5)$ & $3 / 42(7.1)$ & NA \\
\hline LV invasion $(\%)$ & 17/971 (1.8) & $16 / 873(1.8)$ & $1 / 56(1.8)$ & $0(0.0)$ & NA \\
\hline
\end{tabular}

IM intestinal metaplasia, LV lymphovascular, NA not applicable, SM submucosal

a The standard deviation is given in parentheses.

${ }^{a}$ Each denominator is the valid number for the variable. Some of them are less than the total number for each group, because of missing data. Especially for atrophy, many are missing data because of superficial biopsy samples that were inadequate for gland evaluation.

b Among the three groups

neoplasm had developed in $3.0 \%$, and in $2.2 \%$ among those with no eradication, which was not statistically significant. $(p=0.570)$. The time interval between the primary procedure and metachronous tumor development was also not different between the $H$. pylori eradication group and the noneradication group (29.9 months vs 31.3 months, $p=0.893$ ). Kaplan-Meier analysis of the cumulative incidence of metachronous tumor showed no significant difference between the two groups with respect to eradication ( $p=0.620$ ) (Fig. 2).

For synchronous neoplasm, compared with single neoplasm, univariate analysis showed that carcinoma, primary tumor size less than $1 \mathrm{~cm}$, and intestinal metaplasia were positively related, and tumor size less than $1 \mathrm{~cm}$ was found to be the only independent risk factor in multivariate analysis (Table 4). For metachronous neoplasm, compared with single neoplasm, univariate analysis showed that age over 65 years, male gender, absence of $H$. pylori infection, lower third location, and intestinal metaplasia were positively related factors. In the multivariate analysis, the absence of $H$. pylori infection was revealed as the independent risk factor and the lower third location showed near significance (Table 5).

\section{Stratification of follow-up duration}

Because those with longer follow-up duration showed a tendency of more frequent development of multiple gastric neoplasms, subgroup analyses were performed with stratification by 4 years of follow-up. The cut-off of 4 years was taken because with this cut-off the most significant difference in the development of multiple gastric neoplasms was obtained. Among the 671 patients who had been followed up for less than 4 years, univariate analysis demonstrated that age over 65 years, absence of $H$. pylori infection, intestinal metaplasia, and submucosal invasion were associated with multiple neoplasms, and none of these were independently related to multiple neoplasms in multivariate analysis during the first 4 years after the detection of the primary lesion. In this analysis, only intestinal metaplasia showed near significance (OR 4.087, $95 \%$ confidence interval $0.966-17.292 ; p=0.056$ ). In univariate analysis among the 300 patients who had been followed up for 4 years or more, age over 65 years, male gender, absence of $H$. pylori infection, tumor size less than $1 \mathrm{~cm}$, lower third location, intestinal metaplasia, and undifferentiated histologic appearance were shown to be related to multiple neoplasms. However, multivariate analysis showed that lower third location was the only independent risk factor for multiple neoplasms among patients with more than 4 years of follow-up (OR 2.425, $95 \%$ confidence interval 1.148-5.122; $p=0.020$ ).

Characteristics of primary and synchronous or metachronous lesions

In terms of the characteristics of synchronous or metachronous lesions, $60 \%$ were located in the same third of the stomach and $72 \%$ had the same histologic finding of 
Table 2 Univariate analysis for multiple neoplasms

\begin{tabular}{|c|c|c|c|c|}
\hline & $\begin{array}{l}\text { Single } \\
\text { neoplasm } \\
(n=873)^{\mathrm{a}}\end{array}$ & $\begin{array}{l}\text { Multiple } \\
\text { neoplasms } \\
(n=98)^{\mathrm{a}}\end{array}$ & OR & $p$ \\
\hline $\begin{array}{l}\text { Age } \geq 65 \text { years } \\
(\%)\end{array}$ & $402 / 873(46.0)$ & $55 / 98(56.1)$ & 1.488 & 0.058 \\
\hline Male gender (\%) & $640 / 873(73.3)$ & $81 / 98$ (82.7) & 1.735 & 0.045 \\
\hline $\begin{array}{l}\text { Helicobacter } \\
\text { pylori positive } \\
(\%)\end{array}$ & $471 / 838(56.2)$ & 41/95 (43.2) & 0.682 & 0.015 \\
\hline Carcinoma (\%) & $704 / 873(80.6)$ & 83/98 (84.7) & 1.328 & 0.332 \\
\hline Size $\geq 1 \mathrm{~cm}(\%)$ & $691 / 872(79.2)$ & 73/97 (75.3) & 0.787 & 0.362 \\
\hline $\begin{array}{l}\text { Lower third } \\
\text { location }(\%)\end{array}$ & $524 / 873(60.0)$ & 68/98 (69.4) & 1.610 & 0.072 \\
\hline Atrophy (\%) & $470 / 683(68.8)$ & $54 / 70(77.1)$ & 1.680 & 0.149 \\
\hline $\mathrm{IM}(\%)$ & $727 / 837$ (86.9) & 92/95 (96.8) & 4.840 & 0.005 \\
\hline $\begin{array}{l}\text { Undifferentiated } \\
(\%)\end{array}$ & $87 / 872(10.0)$ & $6 / 98(6.1)$ & 0.688 & 0.219 \\
\hline $\mathrm{SM}$ invasion $(\%)$ & $75 / 872(8.6)$ & 10/98 (10.2) & 1.208 & 0.595 \\
\hline LV invasion (\%) & $16 / 873(1.8)$ & $1 / 98(1.0)$ & 0.652 & 1.000 \\
\hline
\end{tabular}

$I M$ intestinal metaplasia, $L V$ lymphovascular, $O R$ odds ratio, $S M$ submucosal

${ }^{a}$ Each denominator is the valid number for the variable.

Table 3 Multivariate analysis for multiple neoplasms

\begin{tabular}{llll}
\hline & OR & $95 \%$ CI & $p$ \\
\hline Age $\geq$ 65 years & 1.429 & $0.924-2.211$ & 0.108 \\
Male gender & 1.547 & $0.889-2.692$ & 0.123 \\
Absence of Helicobacter pylori & 1.610 & $1.038-2.497$ & 0.033 \\
Lower third location & 1.610 & $1.008-2.569$ & 0.046 \\
IM & 4.321 & $1.338-13.957$ & 0.014 \\
\hline
\end{tabular}

CI confidence interval, IM intestinal metaplasia, $O R$ odds ratio

dysplasia or carcinoma as the primary lesions. However, the level of tumor differentiation was same in only $35 \%$ of the synchronous or metachronous cancers.

\section{Discussion}

Although surgical resection has been considered as the only curative treatment for gastric neoplasm, nowadays endoscopic resection is also widely used. Moreover, as more and more gastric cancers are diagnosed at early stages rather than at advanced stages by the national cancer screening program in Korea, the role of endoscopic resection is growing explosively. However, since endoscopic resection spares a large area of gastric mucosa, the later development of synchronous or metachronous neoplasms in another site from the primary lesion is one of the main concerns with this innovative procedure. So far,

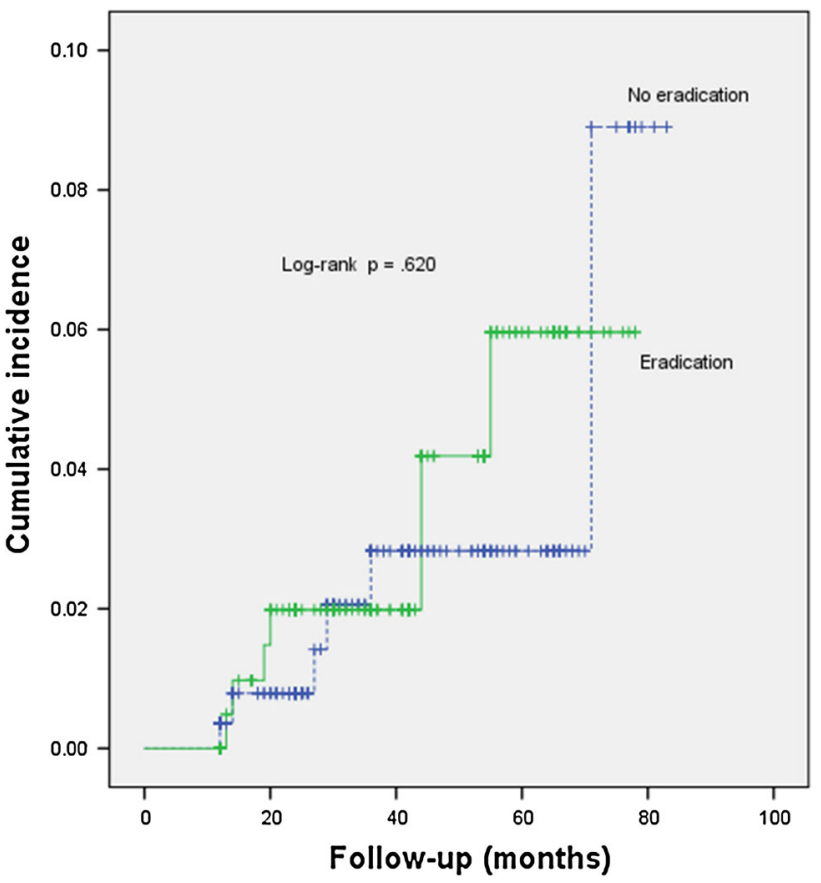

Fig. 2 Kaplan-Meier analysis of cumulative incidence of metachronous tumor with respect to eradication therapy

Table 4 Subgroup multivariate analysis for synchronous neoplasms compared with single neoplasm

\begin{tabular}{llll}
\hline & OR & $95 \%$ CI & $p$ \\
\hline Carcinoma & 2.014 & $0.842-4.817$ & 0.116 \\
Size $<1 \mathrm{~cm}$ & 1.917 & $1.060-3.469$ & 0.031 \\
IM & 3.933 & $0.942-16.418$ & 0.060 \\
\hline
\end{tabular}

CI confidence interval, $I M$ intestinal metaplasia, $O R$ odds ratio

Table 5 Subgroup multivariate analysis for metachronous neoplasms compared with single neoplasm

\begin{tabular}{llll}
\hline & OR & $95 \%$ CI & $p$ \\
\hline Age $\geq$ 65 years & 1.886 & $0.967-3.680$ & 0.063 \\
Male gender & 2.302 & $0.882-6.006$ & 0.089 \\
Absence of Helicobacter pylori & 2.454 & $1.233-4.885$ & 0.011 \\
Lower third location & 2.093 & $1.001-4.379$ & 0.050 \\
IM & 5.236 & $0.706-38.817$ & 0.105
\end{tabular}

$C I$ confidence interval, IM intestinal metaplasia, $O R$ odds ratio

definite risk factors for these multiple gastric neoplasms have not yet been established.

Previously, a few studies suggested risk factors for metachronous cancer, such as H. pylori infection [17], chronic atrophic gastritis [13, 14, 16], and undifferentiated histologic appearance [15]. However, there have been many limitations in terms of relatively small numbers of patients, lack of evaluation for premalignant dysplastic 
lesions, and adjustment with multiple factors. For these reasons, this study was designed to clarify the independent risk factors for synchronous or metachronous gastric neoplasms, including high-grade dysplasia and EGC with a large volume.

In this study, less than $10 \%$ of subjects developed either synchronous or metachronous neoplasms during the follow-up period of 12-131 months (median 35 months). More than half of these multiple neoplasms were detected within 1 year, showing the importance of short-term follow-up within the first year after the primary procedure. However, almost $20 \%$ of the secondary lesions were also found more than 3 years after endoscopic resection, suggesting that long-term follow-up also cannot be ignored. In a previous study of metachronous gastric cancer after endoscopic resection, the overall incidence was reported to be $8.2 \%$ during an average of 3.1 years of follow-up, and the cumulative 3-year incidence of metachronous gastric cancer was $5.9 \%$ [19]. Another study reported that metachronous gastric cancers had an overall incidence of $14 \%$ during a median of 57 months of follow-up [12], and yet another study showed the incidence of synchronous and metachronous multiple gastric cancers after endoscopic resection to be 14.5 and $8.5 \%$, respectively [15]. As highgrade dysplasia was also included in this study, the rate of synchronous or metachronous tumor development might be lower than that found in previous studies because of the lower invasiveness or multiplicity of dysplastic epithelium than invasive carcinoma. Moreover, most of the lesions with low-grade dysplasia which were found during the study period were also removed by endoscopic resection in this study. Although there have been many debates about the malignant potential of low-grade dysplasia, complete removal of all dysplastic epithelium might reduce the risk of synchronous or metachronous tumor development.

This study has revealed that absence of $H$. pylori infection, lower third location, and intestinal metaplasia are independent risk factors for multiple neoplasms. Specifically, a primary tumor size less than $1 \mathrm{~cm}$ was related to synchronous lesions, and the absence of $H$. pylori infection and lower third location were related to metachronous lesions.

The reason why synchronous lesions were more likely to be small is unclear. However, considering that most of the simultaneous multifocal lesions were in the same third of the stomach, there are chances that some small multifocal lesions combine as they grow, becoming single lesions.

In this study, $H$. pylori infection exhibited an inverse relationship with metachronous lesions. It is hard to discuss the statistical significance because of the large difference among the numbers of patients included in each group. However, one of the possibilities is that there might be many previous infections among the $H$. pylori-negative patients and the past long-term infection might have had more effect on metachronous tumorigenesis than newly developed current infection. The fact that the current prevalence of $H$. pylori infection among the older patients was lower than that of the younger patients suggests this assumption. Moreover, H. pylori eradication therapy was shown to be ineffective in preventing metachronous lesions. For the effect of $H$. pylori eradication on metachronous tumor development, a prospective randomized controlled trial has suggested that $H$. pylori eradication therapy had prophylactic power against metachronous gastric cancer [17], whereas another long-term follow-up study showed that baseline mucosal atrophy, but not $H$. pylori status, was independently related to the development of metachronous gastric cancer [16]. Because the proportion of subjects with intestinal metaplasia was much larger in this study than in the previous randomized controlled trial, it seems $H$. pylori eradication therapy could not reverse neoplastic changes and had no preventive role in the progression to metachronous tumors. Moreover, the mean interval to develop new lesions was shown to be no longer in $H$. pylori-eradicated patients than in persistently $H$. pylori-infected patients, which explains why $H$. pylori eradication therapy has no effect in delaying the dysplastic progress.

Since the follow-up duration is strongly associated with the development of metachronous neoplasm, we performed an additional stratification analysis. Within 4 years after the primary procedures, nothing was revealed to be an independent risk factor for multiple neoplasms; however intestinal metaplasia showed near significance. On the other hand, lower third location was the only risk factor for multiple neoplasms in long-term follow-up. This finding confirms that these variables are still closely related to multiple neoplasms even after adjustment for the follow-up duration.

Although $H$. pylori is well known to be associated with development of atrophic gastritis, intestinal metaplasia, and gastric cancer, there is no clear evidence that intestinal metaplasia is improved by $H$. pylori eradication [20, 21]. It seems that once intestinal metaplasia develops, $H$. pylori eradication cannot prevent malignant progression. Therefore, our results seem to mean that irreversible intestinal metaplasia is more critical than $H$. pylori status in the development of multiple gastric neoplasms.

Similarly to the previous study [12], this study showed that new lesions tend to develop in the same third of the stomach with the same histologic features as the primary ones. Endoscopic resection can be applied as long as the new lesions are detected early enough to meet the criteria for it. In the current study, all the patients with new lesions except for five were treated with endoscopic resections. Among these five, two had carcinoma larger than $2 \mathrm{~cm}$ 
with undifferentiated histologic appearance, another two had muscular invasion, and the other one had two separate new lesions, including one recurred lesion. All five had risk factors such as intestinal metaplasia and lower third locations, and the new lesions were detected during scheduled follow-up. These findings suggest that gastric antral neoplasms with intestinal metaplasia treated with endoscopic resections are likely to develop new lesions during followup.

This study contains the limitations of its retrospective nature. However, it has the advantage of a large number of patients with multiple neoplasms.

In conclusion, this study suggests that patients who had undergone endoscopic resection for gastric neoplasms located in the antrum with intestinal metaplasia tend to develop synchronous or metachronous lesions regardless of H. pylori status. Therefore, for patients with these characteristics, more meticulous endoscopic examination with close follow-up should be considered. Further prospective, randomized studies are necessary to set up a tailored follow-up schedule for those who are at high risk of multiple gastric neoplasms.

Acknowledgment This work was supported by a grant from the Liver Research Institute, Seoul National University College of Medicine.

Conflict of interest Joo Hyun Lim, Sang Gyun Kim, Jeongmin Choi, Jong Pil Im, Joo Sung Kim, and Hyun Chae Jung declare that they have no conflict of interest or financial ties.

\section{References}

1. Oda I, Gotoda T, Hamanaka H, Eguchi T, Saito Y, Matsuda T, et al. Endoscopic submucosal dissection for early gastric cancer: technical feasibility, operation time and complications from a large consecutive series. Dig Endosc. 2005;17:54-8.

2. Chung IK, Lee JH, Lee SH, Kim SJ, Cho JY, Cho WY, et al. Therapeutic outcomes in 1000 cases of endoscopic subucosal dissection for early gastric neoplasms: Korean ESD Study Group multicenter study. Gastrointest Endosc. 2009;69:1228-35.

3. Watanabe K, Ogata S, Kawazoe S, Watanabe K, Koyama T, Shimoda Y, et al. Clinical outcomes of EMR for gastric tumors: histolorical pilot evaluation between endoscopic submucosal dissection and conventional mucosal resection. Gastrointest Endosc. 2006;63:776-82.

4. Moertel CG, Gargen JA, Soule EH. Multiple gastric cancers; review of the literature and study of 42 cases. Gastroenterology. 1957;32:1095-103.

5. Kosaka T, Miwa K, Yonemura Y, Urade M, Ishida T, Takegawa $\mathrm{S}$, et al. A clinicopathologic study on multiple gastric cancers with special reference to distal gastrectomy. Cancer. 1990;65:2602-5.

6. Esaki Y, Hirokawa K, Yamashiro M. Multiple gastric cancers in the aged with special reference to intramucosal cancers. Cancer. 1987;59:560-5.

7. Kodera Y, Yamamura Y, Torii A, Uesaka K, Hirai T, Yasui K, et al. Incidence, diagnosis and significance of multiple gastric cancer. Br J Surg. 1995;82:1540-3.

8. Iwafuchi M, Watanabe H. Synchronous multiple early gastric cancer; study of surgically resected stomach. Nippon Rinsho. 1996;54:1217-23.

9. Misudomi T, Watanabe A, Matsusaka T, Fujinaga Y, Fuchigami T, Iwashita A. A clinicopathological study of synchronous multiple gastric cancer. Br J Surg. 1989;76:237-40.

10. Honmyo U, Misumi A, Murakami A, Haga Y, Akagi M. Clinicopathological analysis of synchronous multiple gastric carcinoma. Eur J Surg Oncol. 1989;15:316-21.

11. Takeda J, Toyonaga A, Koufuji K, Kodama I, Aoyagi K, Yano S, et al. Early gastric cancer in the remnant stomach. Hepatogastroenterology. 1998;45:1907-11.

12. Nasu J, Doi T, Endo H, Nishina T, Hirasaki S, Hyodo I. Characteristics of metachronous multiple early gastric cancers after endoscopic mucosal resection. Endoscopy. 2005;37:990-3.

13. Shiotani A, Uedo N, Iishi H, Yoshiyuki Y, Ishii M, Manabe N, et al. Predictive factors for metachronous gastric cancer in highrisk patients after successful Helicobacter pylori eradication. Digestion. 2008;78:113-9.

14. Han JS, Jang JS, Choi SR, Kwon HC, Kim MC, Jeong JS, et al. A study of metachronous cancer after endsocopic resection of early gastric cancer. Scand J Gastroenterol. 2011;46:1099-104.

15. Seo JH, Park JC, Kim YJ, Shin SK, Lee YC, Lee SK. Undifferentiated histology after endoscopic resection may predict synchronous and metachronous occurrence of early gastric cancer. Digestion. 2010;81:35-42.

16. Maehata Y, Nakamura S, Fujisawa K, Esaki M, Moriyama T, Asano K. Long-term effect of Helicobacter pylori eradication on the development of metachronous gastric cancer after endoscopic resection of early gastric cancer. Gastrointest Endosc. 2012;75:39-46.

17. Fukase K, Kato M, Kikuchi S, Inoue K, Uemura N, Okamoto S, et al. Effect of eradication of Helicobacter pylori on incidence of metachronous gastic carcinoma after endoscopic resection of early gastric cancer: an open-label, randomised controlled trial. Lancet. 2008;372:392-7.

18. Kim WH, Park CK, Kim YB, Kim YW, Kim HG, Bae HI, et al. A standardized pathology report for gastric cancer. Korean J Pathol. 2005;39:106-13.

19. Nakajima T, Oda I, Gotoda T, Hamanaka H, Eguchi T, Yokoi C, et al. Metachronous gastric cancers after endoscopic resection: how effective is annual endoscopic surveillance? Gastric Cancer. 2006;9:93-8.

20. Wang J, Xu L, Shi R, Huang X, Li SW, Huang Z, et al. Gastric atrophy and intestinal metaplasia before and after Helicobacter pylori eradication: a meta-analysis. Digestion. 2011;83:253-60.

21. Leung WK, Lin SR, Ching JY, To KF, Ng EK, Chan FK, et al. Factors predicting progression of gastric intestinal metaplasia: results of a randomized trial on $\mathrm{H}$. pylori eradication. Gut. 2004;53:1244-9. 